\title{
Electrically Tunable Plasmonic Absorber Based On Cu-ITO Subwavelength Grating On SOI At Telecom Wavelength
}

\section{Suresh Kumar Pandey ( $\nabla$ suresh143jig@gmail.com )}

Indian Institute of Technology Indore https://orcid.org/0000-0001-7091-5263

\section{Swati Rajput}

Indian Institute of Technology Indore

Vishal Kaushik

Indian Institute of Technology Indore

Prem Babu

Indian Institute of Technology Indore

Rahul Dev Mishra

Indian Institute of Technology Indore

Mukesh Kumar

Indian Institute of Technology Indore

\section{Research Article}

Keywords: Plasmonic, Cu-ITO grating, SPPs, tunable absorber

Posted Date: February 9th, 2022

DOI: https://doi.org/10.21203/rs.3.rs-1326215/v1

License: (c) (i) This work is licensed under a Creative Commons Attribution 4.0 International License.

Read Full License 


\title{
Electrically Tunable Plasmonic Absorber based on $\mathrm{Cu}-\mathrm{ITO}$ Subwavelength grating on SOI at telecom wavelength
}

\author{
Suresh Kumar Pandey*1, Swati Rajput ${ }^{1}$, Vishal Kaushik ${ }^{1}$, Prem Babu ${ }^{1}$, Rahul Dev Mishra ${ }^{1}$ \\ and Mukesh Kumar ${ }^{1,2}$ \\ ${ }^{I}$ Optoelectronic Nanodevice Research Laboratory, Department of Electrical Engineering, \\ Indian Institute of Technology Indore, Indore, India \\ ${ }^{2}$ Centre for Advanced Electronics, Indian Institute of Technology Indore, Indore, India \\ *Corresponding Author email ID: suresh143jig@gmail.com
}

\begin{abstract}
An electrically controlled optical absorption is numerically proposed in a plasmonic waveguide on silicon-oninsulator (SOI) consisting of copper-Indium tin oxide (ITO) based subwavelength grating at $1.55 \mu \mathrm{m}$ wavelength. The $\mathrm{Cu}-\mathrm{ITO}$ subwavelength grating in form of a discontinuous $\mathrm{Cu}$ layer filled with ITO together with electrically tunable permittivity of ITO provides us with a tunable absorption and efficient guidance of plasmonic mode. An n-type ITO is used which exhibits a significant change in the carrier concentration with the applied voltage resulting in a change in optical absorption at a telecom wavelength of $1.55 \mu \mathrm{m}$. We numerically observe maximum tuning in absorption at a grating period of $600 \mathrm{~nm}$ and a duty cycle of $50 \%$. The proposed device shows a smaller effective mode area of $A m=0.01421 / \mu \mathrm{m} 2$ and a plasmonic confinement factor of 34.67 $\%$. The device is reported to have an extinction ratio of $10.28 \mathrm{~dB}$ for a $100 \mu \mathrm{m}$ long device at a low voltage of $6 \mathrm{~V}$.
\end{abstract}

Index Terms - Plasmonic, Cu-ITO grating, SPPs, tunable absorber

\section{INTRODUCTION}

The absorption of light energy is a important property of light in which the energy of an electromagnetic wave striking on an object is transferred to several usable types of energies, for example thermal, electrical, chemical, and mechanical. These kinds of devices can be used in a variety of ways like photodetection [1], local heating [2], bio-sensing [3-4], imaging [5], and energy harvestings [6-8]. Noble metals such as copper, gold, and silver are excellent plasmonic materials in the infrared regime (IR). By pattering such types of metals at the nanoscale level, strong absorption can be produced due to the excitation of Surface plasmons polaritons (SPPs) [9-15]. For the integration of nanoscale devices on a single chip, the control and confinement of the light beyond the diffraction limit are major challenges that can be overcome by plasmonic [16-19]. Surface plasmon (SP) excitations in metals_allow light to be trapped in the deepsubwavelength zone, which can improve strong light-matter interactions, plasmonic has allowed us to miniaturization of components at a nanoscale level so we can integrate a large number of optical and electronic components on a single chip [23-
24]. Controlling and guiding light in on-chip devices via electrical means is a key technology for high-speed computing and embedding data on an optical carrier for efficient data transmission. In that situation, the electrical tuning of a waveguide remains an important function for a variety of devices that can be generated by electrically tuning the charge carrier density of the material to change the optical parameter of the guided wave [25-28]. The carrier concentration of Transparent Conducting Oxides (TCOs) can be changed to tune the optical properties of light. Among the number of TCOs, Indium Tin Oxide (ITO) is the preferred candidate to tune optical properties. It has received interest as an active electro-optical material because of the significant refractive index change owing to permittivity changes at NIR wavelengths, including the $1.55 \mu \mathrm{m}$ telecommunication wavelength [29,30]. An integrated platform based on Surface Plasmon Polariton (SPPs) waveguide can be promising for making photonic devices at the nanoscale. Plasmonic waveguide structures offers confinement of optical energy by coupling electromagnetic wave to the oscillation of free charge carriers present at metal-dielectric interface called surface plasmon polariton(SPPs). The oscillation wavelength of resulting plasmon polaritons are considerably smaller as compared to the wavelength of light energy in the vacuum [33].

In this paper, an electrically tunable plasmonic absorber on a silicon-on-insulator (SOI) is proposed. The device consists of Copper-Indium Tin Oxide (ITO) based subwavelength grating at $1.55 \mu \mathrm{m}$ wavelength. The slow light propagates in the device arising from light confinement in the small mode area. The effective mode area of $\mathrm{Am}=0.01421 / \mu \mathrm{m} 2$ and plasmonic confinement factor of $34.67 \%$ is observed, resulting in enhanced light-matter interaction and significant change in the imaginary part of effective effective refractive index which shows the absorption of light. The $\mathrm{Cu}$-ITO subwavelength grating in form of a discontinuous $\mathrm{Cu}$ layer filled with ITO together with electrically tunable permittivity of ITO provides us with a tunable absorption and efficient guidance of plasmonic mode. An n-type ITO is used which exhibits a significant change in carrier concentration with the applied voltage of $4 \mathrm{~V}$, resulting in a change in optical absorption at telecom wavelength. In this work the significant change of the imaginary part of the effective effective refractive index with varying the duty cycle at the grating period of $600 \mathrm{~nm}$ and we observe maximum tuning in absorption at a grating period of $600 \mathrm{~nm}$ and a duty cycle of $50 \%$. The propagation length of the device with varying the duty cycle and with increasing the duty 
cycle the propagation length decreases. The device has an extinction ratio of $10.28 \mathrm{~dB}$ for a $100 \mu \mathrm{m}$ long device at a very low voltage of $6 \mathrm{~V}$.

\section{DeVICE STRUCTURE \& WORKING PRINCIPLE}

In the proposed device Indium Tin Oxide (ITO) is used as an active absorber material, light absorption can be controlled electrically, the ITO has established itself as one of the highly electrically controllable material and the optical characteristics of the material can be varied significantly by electrical tuning of free charge carrier [20]. ITO exhibits unity order change of effective refractive index due to change in permittivity of the material which can be used for tunable absorber device [35]. Fig.1 shows the 3-D geometry of proposed of the electrically tunable plasmonic absorber device. Here $\mathrm{x}$-axis depicts the device's width, the y-axis denotes the device's thickness, and the $\mathrm{z}$-axis represents the propagation direction of the electromagnetic wave. The silicon rib waveguide's thickness $(t)$ and width(w) are $200 \mathrm{~nm}$ and $300 \mathrm{~nm}$, respectively, and the device has a buried oxide layer for isolation from the silicon substrate for better confinement of light.

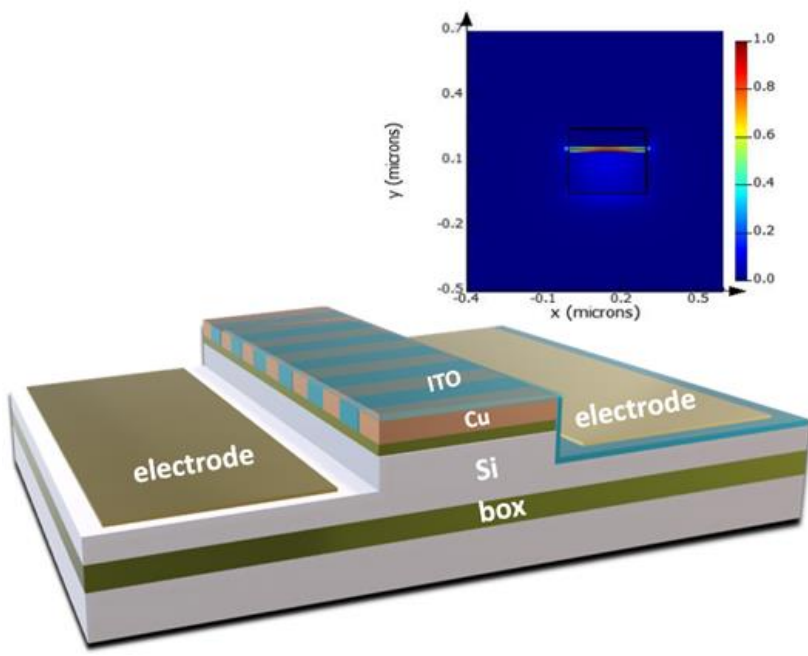

Fig.1 3-D diagram of proposed Cu-ITO subwavelength grating assisted electrically tunable plasmonic absorber at $1550 \mathrm{~nm}$ wavelength on p-type SOI wafer. $\mathrm{Cu}$-ITO subwavelength grating is utilized to change the imaginary part of the effective refractive index $(\kappa)$ of the propagating plasmonic mode of the device. Si rib waveguide width is $300 \mathrm{~nm}$, Si thickness is $200 \mathrm{~nm}, \mathrm{Cu}-\mathrm{ITO}$ grating thickness is $40 \mathrm{~nm}, \mathrm{Cu}-\mathrm{ITO}$ grating period is $600 \mathrm{~nm}$, the Duty cycle is $50 \%$ and ITO capping layer is $40 \mathrm{~nm}$ and Plasmonic mode (TM mode) confinement at dielectric and Cu-ITO grating interface of the device. Inset shows confinement of the guided plasmonic TM mode.

The device consists of a $10 \mathrm{~nm}$ thick silicon dioxide layer over which an alternating $\mathrm{Cu}$ and ITO grating is formed. Distributed $\mathrm{Cu}$ layer is used to reduce metal absorption and to enhance propagation length. ITO material filled between two $\mathrm{Cu}$ strips interact more with SPPs which enhances tuning of carrier concentration ITO layer. The refractive index of $\mathrm{Cu}$ (0.7) and ITO (1.3) at telecom wavelength $(1550 \mathrm{~nm})$ makes a good contrast for the slow light effect which can enhance the light-matter interaction of the device. The grating period of the device is $600 \mathrm{~nm}$ and the duty cycle is $50 \%$. The optimized thickness of both $\mathrm{Cu}$ and ITO grating is $40 \mathrm{~nm}$ each over which a $40 \mathrm{~nm}$ thick capping layer of ITO is used for better electrical tuning of the device. A $10 \mathrm{~nm}$ layer of silicon dioxide dielectric material is used to excite the SPPs at the metal-dielectric interface and to enhance the plasmonic mode. For the simulation of the device Lumerical Eigen Mode, Finite Difference solver is used to study the field confinement of the plasmonic mode and observed that the device supports Transverse Magnetic TM polarized wave propagation and mode confined at the interface of dielectric and $\mathrm{Cu}-\mathrm{ITO}$ grating. The working principle of the device is when we shine the light having the wavelength of 1550 $\mathrm{nm}$, The TM polarized plasmonic mode propagates with a high confinement factor and tiny mode area at the $\mathrm{Cu}$-ITO grating and dielectric interface, the inset shows the mode confinement of the device. the charge carrier accumulates at $\mathrm{Cu}$-ITO grating and dielectric interface, and the accumulated carriers efficiently coupled by propagating electromagnetic waves and form Surface Plasmon Polariton (SPPs) which changes the permittivity of ITO material. The imaginary component of the effective refractive index of the waveguide changes when the permittivity of the ITO changes, indicating a change in light.

\section{ANALYSIS OF EFFECT OF ITO AND PROPAGATION CHARACTERISTICS}

The device's optical properties can be electrically modified by varying the carrier (electron) concentration in the ITO layer. It's a good material for tuning intensity at telecom wavelengths with high modulation efficiency because of the substantial fluctuation in carrier concentration caused by the applied voltage. The mobile electron concentration density of the ITO, which acts as a free electron Drude material, is used to monitor its optical charectiristics[31].

The optical permittivity of the ITO is denoted by

$$
\begin{aligned}
& \varepsilon_{I T O}=\varepsilon_{\infty}-\frac{\omega_{p}^{2}}{\omega^{2}+i \omega \Gamma} \\
& \omega_{p}^{2}=\frac{N_{e}^{2}}{\varepsilon_{0} m_{c e}^{*}}
\end{aligned}
$$

Here $\varepsilon_{\infty}$ represents dielectric constant of the material, $\omega$ depicts the angular frequency of propagating electromagnetic wave and the carrier collision frequency in the ITO layer of the device is denoted by $\Gamma, \omega_{p}$ is the plasma frequency of the ITO layer, $N_{e}$ is electron charge magnitude and $m_{c e}^{*}$ is the effective mass of the electron. The permittivity of the ITO layer can be changed by applying the electrical bias due to the variation of charge carrier density of the ITO layer. Lumerical charge solver is used to analyzing the variation of charge carrier density with applied bias. Fig.2(a) shows the change of carrier with applied voltage at different ITO thickness and Fig.2(b) shows the change of carrier concentration versus vertical distance(thickness) of the waveguide and it is observed that the carrier accumulates at the $\mathrm{Cu}$-ITO grating and $\mathrm{SiO}_{2}$ interface, the accumulated carrier changes the permittivity of the ITO material. A significant change of carrier concentration at the applied voltage of $4 \mathrm{~V}$ is observed. The change of carrier concentration results in changes in the effective refractive index of the material due to a change of permittivity of the ITO layer. The perturbed carrier concentration of Lumerical charge solver due to 
(a)

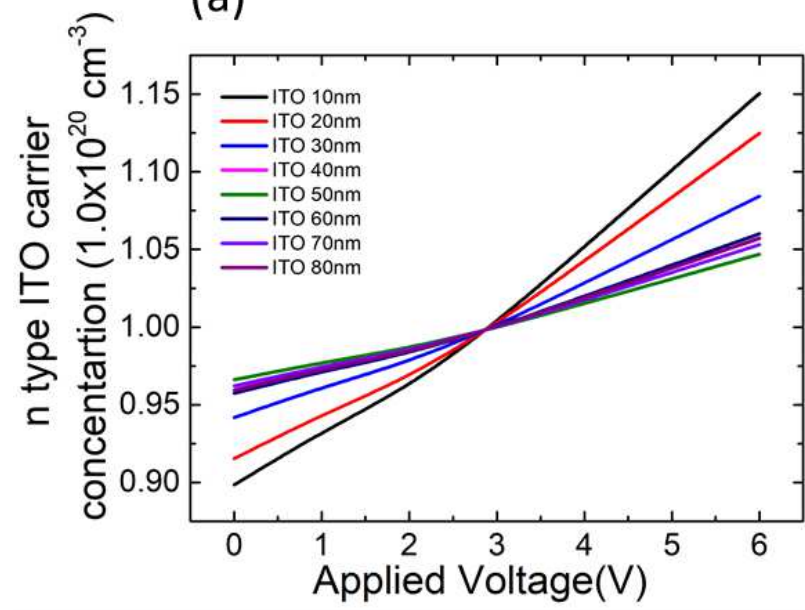

(b)

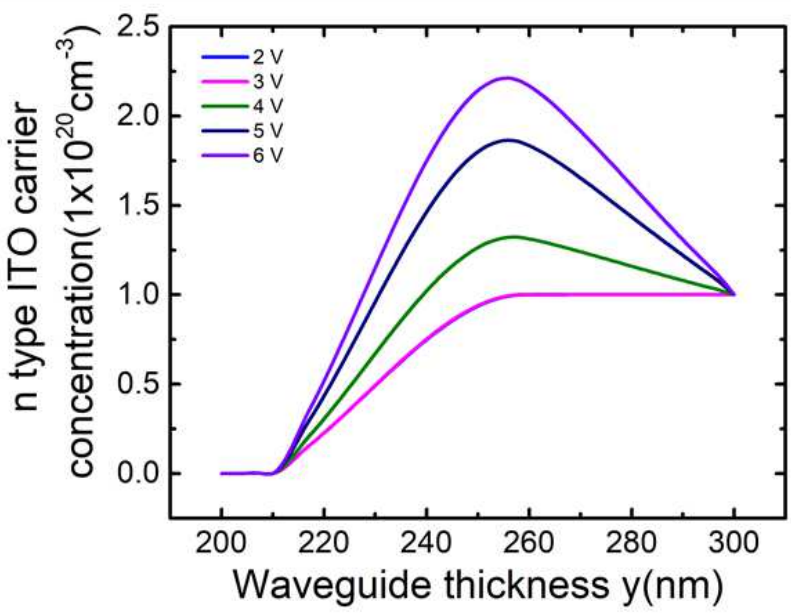

Fig.2(a) change of carrier concentration with varying the thickness of ITO layer at different bias voltage, the horizontal axis shows the thickness of waveguide in $\mathrm{nm}$. (b) change of carrier concentration with applied voltage at ITO $\left(1 \times 10^{20} \mathrm{~cm}^{-3}\right)$ thickness of $40 \mathrm{~nm}$. The device exhibits an abrupt change of carrier concentration at $4 \mathrm{~V}$.

applied bias is imported in Lumerical Finite Difference Time Domain (FDTD) to observe the change of wave propagation. Fig3(a) shows the wave propagation, at no bias the plasmonic wave propagate at the interface of dielectric and $\mathrm{Cu}$-ITO grating and we can say that $\mathrm{ON}$ state of the device and when we use perturbed carrier concentration at an applied bias of $4 \mathrm{~V}$, the intensity of wave propagation reduces and device act as an absorber as Shown in Fig.3(b) and we can say OFF state of the device. In the simulation of the device, we have used the Lumerical Finite Difference Eigen Mode (FDE) solver for the analysis of mode confinement.

The effective mode area of the guided mode will be the measure of the nature, whether the localized field is optical or plasmonic mode and it would be the merit of confinement ability, the effective mode area $\left(\mathrm{A}_{\mathrm{m}}\right)$ of the device can be calculated by taking the ratio of total mode energy flux density, and maximum energy flux density at active region. The effective mode area is denoted by [22]

$$
A m(/ \mu m 2)=\int_{-\infty}^{\infty} P(x, y) d x d y / \max [P(x, \gamma)]
$$

Fig.4(a) shows the variation of effective mode area with increasing the thickness of the ITO layer and light confined at a very small mode area of $0.01421 / \mu \mathrm{m}^{2}$ at the active region of the device.
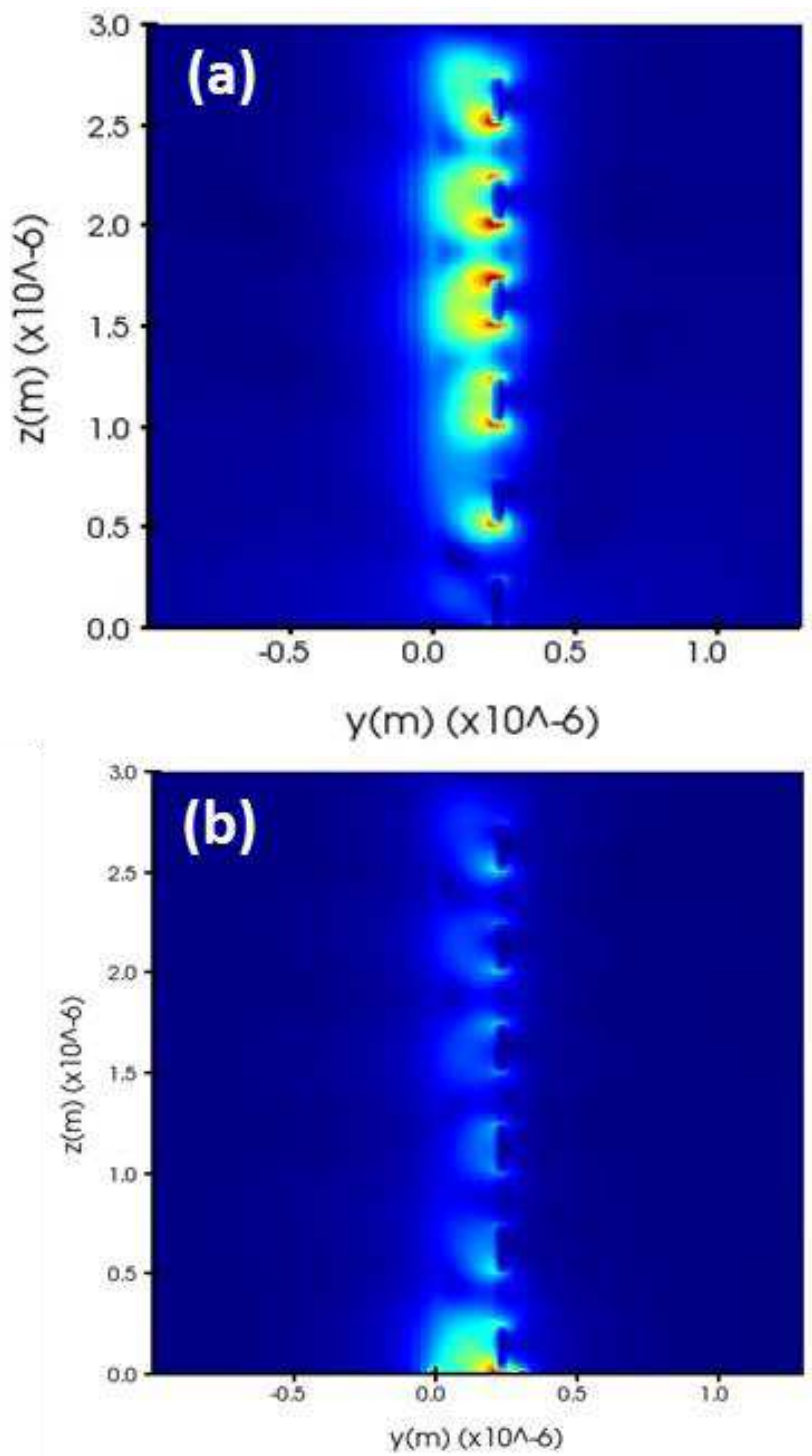

Fig 3 (a) plasmonic wave propagation of the device when no bias is applied (ON state), $\mathrm{y}$ and $\mathrm{z}$ axes represent waveguide thickness and direction of wave propagation respectively and (b) Plasmonic wave propagation at an applied voltage of $4 \mathrm{~V}$ (OFF state).

The propagation length of the plasmonic wave is depicted as the distance at which the intensity of the propagating wave reduce to a factor of $1 / \mathrm{e}$ of the total coupling intensity of the light and the propagation length of the guided plasmonic mode is denoted by [21]

$$
L_{m}(\mu m)=\frac{\lambda}{4 \pi\left(\left(n_{e f f}\right) i m g\right)}
$$

Where $\lambda$ is operating wavelength and $\left(n_{\text {eff }}\right) i m g$ is an imaginary component of the effective refractive index and the model propagation loss is given by

$l_{m}(d B / \mu m)=-\left(2\left(k_{0}\right) \times\left(n_{e f f}\right) i m g\right) \times 4.34$.

where $k_{0}=\frac{2 \pi}{\lambda_{0}}$ denotes the propagation constant of the plasmonic mode and the negative sign indicates lossy materials. 

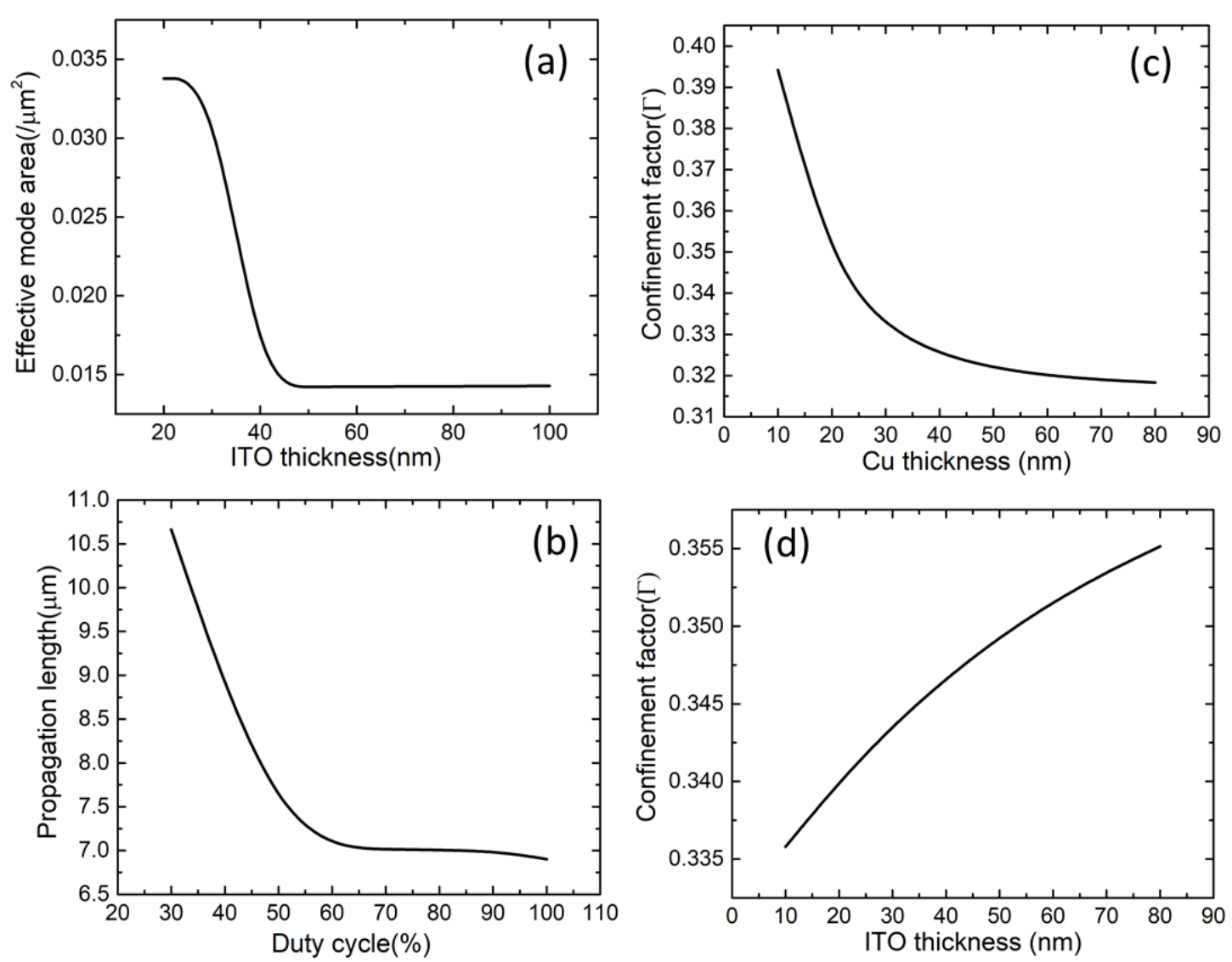

Fig.4(a) effective node area(Am) verses the thickness of $\mathrm{Cu}$ layer, mode area increases with increasing the thickness of $\mathrm{Cu}$ layer.(b) Propagation length of the device with varying the duty cycle, (c) Plasmonic mode confinement at the different thickness of ITO layer of the device, confinement factor increases with increasing the thickness of ITO layer. (d) Plasmonic mode confinement at the different thickness of Cu layer of the device

Fig.4(b) shows the propagation length versus duty cycle of $\mathrm{Cu}$-ITO grating and the propagation length decreases by increasing the duty cycle of grating, this is due to the high model propagation loss of the device. So, there is a tradeoff between model propagation loss and propagation length.

The optical energy confinement of the device is the propagation of power flowing through the active zone to the total power flowing through the system [32]. Fig.4(c) shows the plasmonic mode confinement of the device with varying the thickness of the active layer (ITO) and the confinement factor increases with increasing the thickness of the ITO layer and Fig.4(d) depicts the device's plasmonic mode confinement as a function of $\mathrm{Cu}$ layer thickness, demonstrating the mode confinement decreases as $\mathrm{Cu}$ layer thickness increases. So, the device exhibits the confinement factor of $34.67 \%$ at the optimized layer of $40 \mathrm{~nm}$ of the copper layer and $40 \mathrm{~nm}$ of the ITO layer.

\section{ELECTRICALLY TUNABLE ABSORPTION}

The electrical behavior of the device is numerically investigated by using a Lumerical charge transport solver. The device simulation yields charge transport data of change of charge carrier density of $\mathrm{Cu}$-ITO junction with applied bias. The perturbed charge carrier density of the device is introduced from the Lumerical Device simulation tool through the index perturbation into the Lumerical mode solution for analysis of change of light properties due to applied bias. The Drude model is used to calculate the change in the effective refractive index of the Cu-ITO subwavelength grating. The change of the charge carrier density of the ITO at different voltages has ability to change the effective refractive index of the device. The complex permittivity of the material is related to its charge carrier density, according to the Drude model hypothesis and depicted as $\Delta n=$ $\frac{\Delta \varepsilon}{2 \sqrt{\varepsilon}}$, the variation of charge carrier density(accumulation or deplition) has ability to change the complex permittivity which yields the change of effective refractive index. The change of complex effective refractive index(real and imaginary components) of the device with variation of carrier density is calculated as

$\Delta n=\frac{-e^{2} \lambda_{0}^{2}}{8 \pi^{2} c^{2} \varepsilon_{0} n}\left(\frac{N e}{m_{c}^{*} e}+\frac{N_{h}}{m_{c}^{*} \cdot h}\right)$

and

$\Delta \alpha=\frac{e^{3} \lambda_{0}^{2}}{4 \pi^{2} c^{3} \varepsilon_{0} n}\left(\frac{N e}{\mu_{e}\left(m_{c e}^{*}\right)^{2}}+\frac{N_{h}}{\mu_{h}\left(m_{c h}^{*}\right)^{2}}\right)$

Here, $N_{e}$ and $N_{h}$ represent electron and hole carrier densities, e represents electronic charge, $\lambda_{0}$ represents operation wavelength, $\mu_{e}$ and $\mu_{h}$ represent electron and hole mobility, and $m_{c e}^{*}$ and $m_{c h}^{*}$ represent the effective mass of electron and hole, respectively.

The carrier concentration of the ITO layer is $1 \times 10^{20} \mathrm{~cm}^{-3}$ and when we applied the voltage carrier concentration of the ITO changes as shown in Fig. 2. and electron accumulated at the interface of dielectric and ITO which alters the permittivity of the material yields change in the refractive index of the device when no voltage is applied the refractive index of the material is $2.402+0.0349 \mathrm{i}$ and when we applied voltage of $6 \mathrm{~V}$, the refractive index of the material changed to $2.335+0.048 \mathrm{i}$ at the operating wavelength of $1550 \mathrm{~nm}$. The change in carrier concentration (accumulation of electron at $\mathrm{Cu}$-ITO grating and dielectric interface) due to applied bias, 
(a)

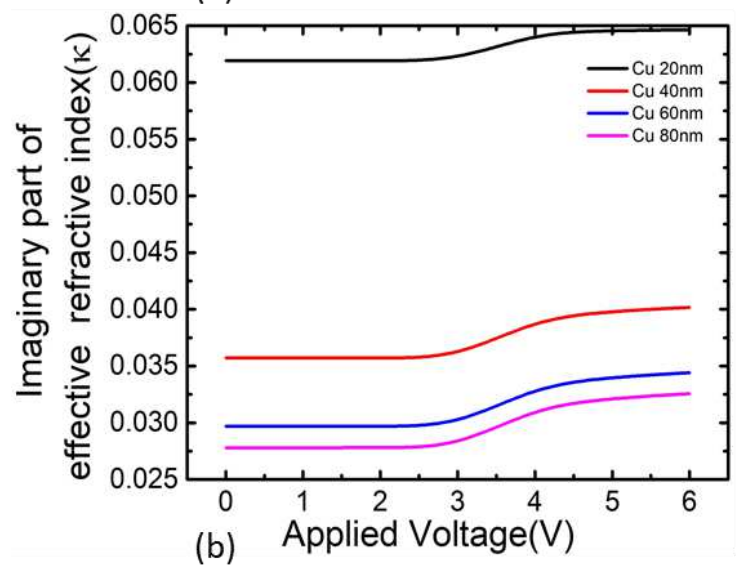

(b) Applied Voltage(V)

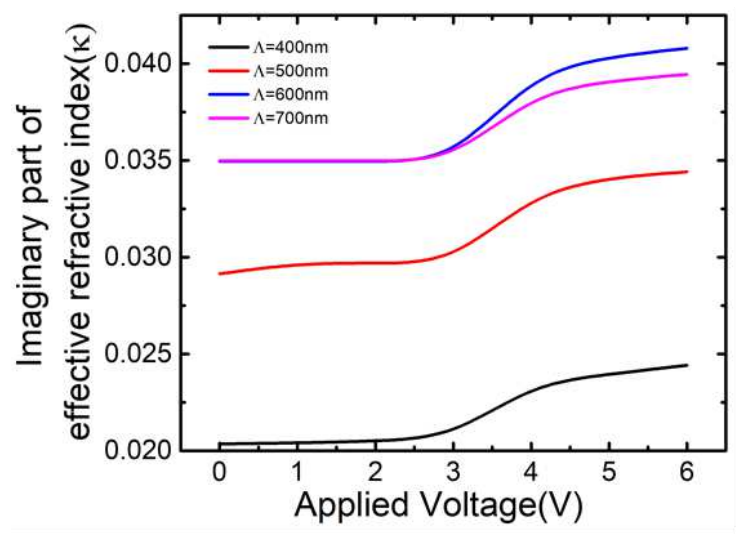

(c)

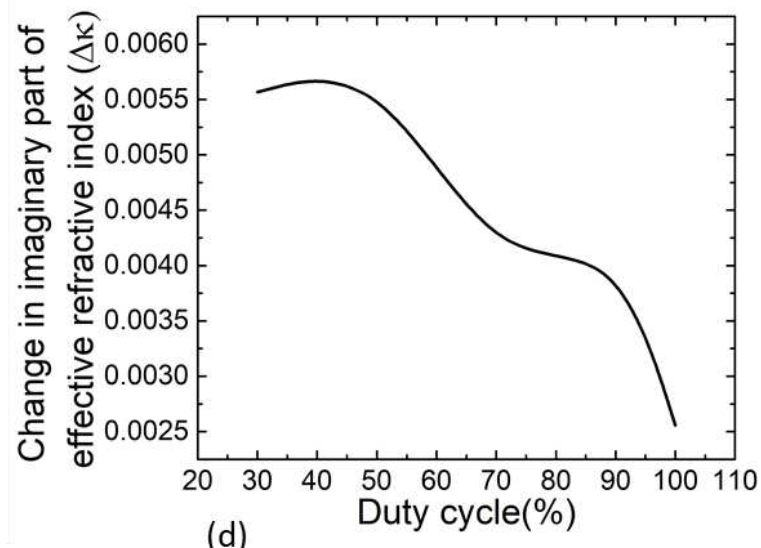

(d)

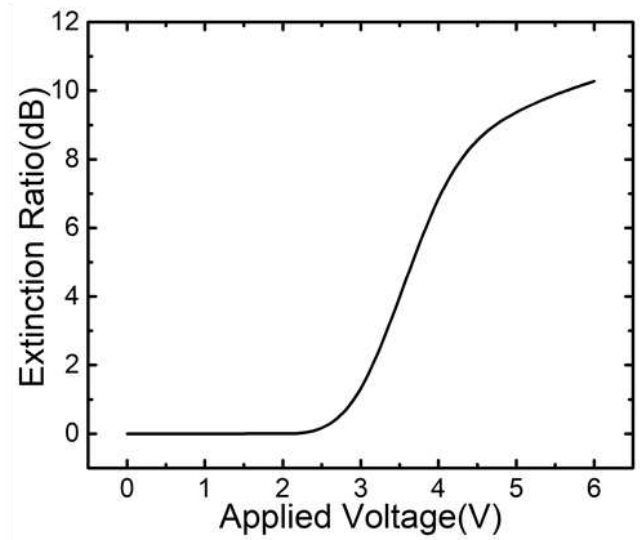

Fig.6(a) imaginary component of effective refractive index with applied voltage at different $\mathrm{Cu}$ thickness of the device. (b)the variation of imaginary component of the refractive index with applied bias at varying the duty cycle of the grating. (c) the imaginary component of the refractive index with applied voltage at varying the grating period of the device, maximum tunability of the absorption(in terms of change in effective refractive index is observed at $600 \mathrm{~nm}$ grating period. (d)The difference in the states of high and low absorptions i.e., Extinction ratio, versus applied voltage. The device yield extinction ratio of $10.28 \mathrm{~dB}$ at the applied voltage of $6 \mathrm{~V}$ for $100 \mu \mathrm{m}$ long device

there is a change of real and imaginary components of the effective index of the device. The change of the real part represents the phase shift and the change of the imaginary part represents the attenuation of plasmonic wave propagation, The electromagnetic wave's intensity can be changed by changing the imaginary components, which can be done with the applied voltage. Fig. 6(a) shows the graph between change in the imaginary component of effective refractive index versus applied voltage with varying the thickness of the $\mathrm{Cu}$ layer of the device and it shows that the imaginary component of the effective refractive index increases with decreasing the thickness of the $\mathrm{Cu}$ layer. We have observed that the maximum tunability of the device is achived at copper and ITO thickness of $40 \mathrm{~nm}$ each. we have optimized the grating period and duty cycle of the device to achieve higher tunability of the plasmonic wave and in Fig.6(b) shows the graph between the change of imaginary component of the effective refractive index with varying the grating period of the device. The change of the imaginary component of the effective refractive index is maximum at the grating period of $600 \mathrm{~nm}$ and Fig.6(c) depicts the variation of the imaginary component of the effective refractive index versus the duty cycle of $\mathrm{Cu}-\mathrm{ITO}$ grating and device yield the maximum tunability at the Duty cycle of 50\%. We optimized the device for an electrically tunable absorber and observe that at the grating period of $600 \mathrm{~nm}$ and duty cycle of $50 \%$, the device yields a maximum change in effective refractive index which represents absorption of electromagnetic wave. The change in the imaginary component of the effective refractive index of the device can be used to tune the attenuation of the intensity (absorption) of the electromagnetic wave. We can calculate the absorption of the light in terms of effective refractive index is given by $\alpha=\frac{2 \pi k_{e f f}}{\lambda}$. The extinction ratio or modulation depth of the device can be calculated by

$E R=4.343[\alpha(V)-\alpha(0)] L$.

Fig. 6(d) shows the extinction ratio versus voltage. The ER of the $100 \mu \mathrm{m}$ long device is calculated to be $10.28 \mathrm{~dB}$. The device length must be increased or a greater voltage must be provided to improve the extinction ratio. The higher driving voltage is incompatible with CMOS driving circuits, and rising device length is incompatible with high-density integration, therefore there is a tradeoff between high extinction ratio and small device footprint.

\section{CONCLUSION}

An electrically tunable plasmonic absorber on SOI is numerically reported. The device consists of copper- Indium tin oxide (ITO) based subwavelength grating which operates at a telecom wavelength of $1.55 \mu \mathrm{m}$. The device design with $\mathrm{Cu}$-ITO structure efficiently utilizes electrically tunable permittivity of ITO. We observe that maximum tuning of absorption at a grating period of $600 \mathrm{~nm}$ and $50 \%$ duty cycle of the Cu-ITO grating. The device shows a very small mode area of $A_{m}=0.01421 / \mu m^{2}$ and a higher plasmonic confinement factor of $34.67 \%$. our device exhibits the Extinction Ratio (ER) of $10.28 \mathrm{~dB}$ at a very low voltage of $6 \mathrm{~V}$ for 
a $100 \mu \mathrm{m}$ long device. This tunable absorber can be used in many applications where attenuation of intensity is a prime requirement such as intensity modulator, biosensing, imaging, etc.

Authors' contributions. S.K.Pandey and M. Kumar conceptualized the device and conducted a theoretical analysis.Numerical analysis was carried out by S.K.Pandey, S. Rajput. V.Kausik, P.Babu and R.D. Mishra provided partial technical suggestions and help in finalizing the manuscript.M. Kumar guided the entire computation process. S.K.Pandey and M.Kumar conducted the data analysis and wrote the manuscript.

Funding. Department of Science and Engineering Research Board (SERB), Ministry of Science and Technology, India (CRG/2020/000144); Council of Scientific and Industrial Research, India (22/0840/20/EMR-II).

Disclosures. The authors declare no conflicts of interest.

Data availability. The data sets generated during and/or analyzed during the current study are available from the corresponding author on reasonable request.

Code availability. The computer codes used during the current study are available from the corresponding author on reasonable request.

\section{Declarations}

Ethics Approval I have read Plasmonic's Ethical Guidelines and state that I have not violated them in any way.

Consent to Participate Informed consent was obtained from all individual participants included in the study.

\section{Consent of Publication Not applicable.}

Conflict of Interest The authors declare no competing interests. I would like to declare on behaf of my co-authors that the work described was original research that has not been published previously, and not under consideration for publication elsewhere, in whole or in part. All the authors listed have approved the manuscript that is enclosed.

\section{REFERENCES}

[1] Cao, L., White, J.S., Park, J.S., Schuller, J.A., Clemens, B.M. and Brongersma, M.L., 2009. Engineering light absorption in semiconductor nanowire devices. Nature materials, $8(8)$, pp.643-647, https://doi.org/10.1038/nmat2477

[2] Baffou, G. and Quidant, R., 2013. Thermo-plasmonics: using metallic nanostructures as nano-sources of heat. Laser \& Photonics Reviews, 7(2), pp.171-187, https://doi.org/10.1002//por.201200003

[3] Anker, J.N., Hall, W.P., Lyandres, O., Shah, N.C., Zhao, J. and Van Duyne, R.P., 2010. Biosensing with plasmonic nanosensors. Nanoscience and Technology: A Collection of Reviews from Nature Journals, pp.308319, https://doi.org/10.1142/9789814287005_0032

[4] Adato, R. and Altug, H., 2013. In-situ ultra-sensitive infrared absorption spectroscopy of biomolecule interactions in real time with plasmonic nanoantennas. Nature communications, $4(1), \quad$ pp.1-10, https://doi.org/10.1038/ncomms3154
[5] Zhang, R., Zhang, Y., Dong, Z.C., Jiang, S., Zhang, C., Chen, L.G., Zhang, L., Liao, Y., Aizpurua, J., Luo, Y.E. and Yang, J.L., 2013. Chemical mapping of a single molecule by plasmon-enhanced Raman scattering. Nature, 498(7452), https://doi.org/10.1038/nature12151

[6] Atwater, H.A. and Polman, A., 2011. Plasmonics for improved photovoltaic devices. Materials for sustainable energy: a collection of peer-reviewed research and review articles from Nature Publishing Group, pp.1-11, https://doi.org/10.1142/9789814317665_0001

[7] Hägglund, C., Zeltzer, G., Ruiz, R., Thomann, I., Lee, H.B.R., Brongersma, M.L. and Bent, S.F., 2013. Self-assembly based plasmonic arrays tuned by atomic layer deposition for extreme visible light absorption. Nano letters, 13(7), pp.3352-3357, https://doi.org/10.1021/n1401641v

[8] Kim, S.J., Thomann, I., Park, J., Kang, J.H., Vasudev, A.P. and Brongersma, M.L., 2014. Light trapping for solar fuel generation with Mie resonances. Nano letters, 14(3), pp.1446-1452, https://doi.org/10.1021/n1404575e

[9] Landy, N.I., Sajuyigbe, S., Mock, J.J., Smith, D.R. and Padilla, W.J., 2008. Perfect metamaterial absorber. Physical review letters, 100(20), p.207402, https://doi.org/10.1103/PhysRevLett.100.207402

[10] Liu, N., Mesch, M., Weiss, T., Hentschel, M. and Giessen, H., 2010. Infrared perfect absorber and its application as plasmonic sensor. Nano letters, 10(7), pp.2342-2348, https://doi.org/10.1021/n19041033

[11] Zhou, H., Ding, F., Jin, Y. and He, S., 2011. Terahertz metamaterial modulators based on absorption. Progress In Electromagnetics Research, 119, pp.449-460, doi:10.2528/PIER11061304

[12] Zhang, B., Zhao, Y., Hao, Q., Kiraly, B., Khoo, I.C., Chen, S. and Huang, T.J., 2011. Polarization-independent dual-band infrared perfect absorber based on a metal-dielectric-metal elliptical nanodisk array. Optics express, 19(16), pp.15221-15228, https://doi.org/10.1364/OE.19.015221

[13] Aydin, K., Ferry, V.E., Briggs, R.M. and Atwater, H.A., 2011. Broadband polarization-independent resonant light absorption using ultrathin plasmonic super absorbers. Nature communications, 2(1), pp.1-7, https://doi.org/10.1038/ncomms1528

[14] Hendrickson, J., Guo, J., Zhang, B., Buchwald, W. and Soref, R., 2012. Wideband perfect light absorber at midwave infrared using multiplexed metal structures. Optics letters, 37(3), pp.371-373, https://doi.org/10.1364/OL.37.000371

[15] Cui, Y., Fung, K.H., Xu, J., Ma, H., Jin, Y., He, S. and Fang, N.X., 2012. Ultrabroadband light absorption by a sawtooth anisotropic metamaterial slab. Nano letters, 12(3), pp.1443-1447, https://doi.org/10.1021/nl204118h

[16] Feng, N.N., Brongersma, M.L. and Dal Negro, L., 2007. Metal-dielectric slot-waveguide structures for the propagation of surface plasmon polaritons at $1.55 \$ \mathrm{mu}\{$ \hbox $\{\mathrm{m}\}\}$ \$. IEEE Journal of Quantum Electronics, 43(6), pp.479-485, 10.1109/JQE.2007.897913

[17] Bian, Y. and Gong, Q., 2014. Bow-tie hybrid plasmonic waveguides. Journal of Lightwave Technology, 32(23), pp.4504-4509.

[18] Grandidier, J., Des Francs, G.C., Massenot, S., Bouhelier, A., Markey, L., Weeber, J.C., Finot, C. and Dereux, A., 2009. Gain-assisted propagation in a plasmonic waveguide at telecom wavelength. Nano letters, 9(8), pp.29352939, https://doi.org/10.1021/nl901314u

[19] Oulton, R.F., Sorger, V.J., Genov, D.A., Pile, D.F.P. and Zhang, X., 2008. A hybrid plasmonic waveguide for subwavelength confinement and longrange propagation. nature photonics, 2(8), pp.496-500, https://doi.org/10.1038/nphoton.2008.131

[20] Feigenbaum, E., Diest, K. and Atwater, H.A., 2010. Unity-order index change in transparent conducting oxides at visible frequencies. Nano letters, 10(6), pp.2111-2116, https://doi.org/10.1021/n11006307

[21] Kou, Y., Ye, F. and Chen, X., 2011. Low-loss hybrid plasmonic waveguide for compact and high-efficient photonic integration. Optics express, 19(12), pp.11746-11752, https://doi.org/10.1364/OE.19.011746

[22] Gao, L., Tang, L., Hu, F., Guo, R., Wang, X. and Zhou, Z., 2012. Active metal strip hybrid plasmonic waveguide with low critical material gain. Optics express, 20(10), pp.11487-11495, https://doi.org/10.1364/OE.20.011487

[23] Schuller, J.A., Barnard, E.S., Cai, W., Jun, Y.C., White, J.S. and Brongersma, M.L., 2010. Plasmonics for extreme light concentration and manipulation. Nature materials, 9(3), pp.193-204, https://doi.org/10.1038/nmat2630

[24] Rajput, S., Kaushik, V., Jain, S., Tiwari, P., Srivastava, A.K. and Kumar, M., 2019. Optical modulation in hybrid waveguide based on Si-ITO heterojunction. Journal of Lightwave Technology, 38(6), pp.1365-1371, $\underline{10.1109 / J L T .2019 .2953690}$ 
[25] Png, C.E., Chan, S.P., Lim, S.T. and Reed, G.T., 2004. Optical phase modulators for $\mathrm{MHz}$ and $\mathrm{GHz}$ modulation in silicon-on-insulator (SOI). Journal of lightwave technology,22(6), p.1573, $\underline{10.1109 / J L T .2004 .827655}$

[26] Tang, C.K. and Reed, G.T., 1995. Highly efficient optical phase modulator in SOI waveguides. Electronics letters, 31(6), pp.451-452.

[27] Jain, S., Rajput, S., Kaushik, V. and Kumar, M., 2019. High speed optical modulator based on silicon slotted-rib waveguide. Optics Communications, 434, pp.49-53, https://doi.org/10.1016/j.optcom.2018.10.028

[28] Tang, C.K., Reed, G.T., Walton, A.J. and Rickman, A.G., 1994. Low-loss, single-model optical phase modulator in SIMOX material. Journal of lightwave technology, 12(8), pp.1394-1400, 10.1109/50.317527

[29] Park, J., Kang, J.H., Kim, S.J., Liu, X. and Brongersma, M.L., 2017. Dynamic reflection phase and polarization control in metasurfaces. Nano letters, 17(1), pp.407-413, https://doi.org/10.1021/acs.nanolett.6b04378

[30] Huang, Y.W., Lee, H.W.H., Sokhoyan, R., Pala, R.A., Thyagarajan, K., Han, S., Tsai, D.P. and Atwater, H.A., 2016. Gate-tunable conducting oxide metasurfaces. Nano letters, 16(9), pp.5319-5325, https://doi.org/10.1021/acs.nanolett.6b00555

[31] Rajput, S., Kaushik, V., Babu, P., Tiwari, P., Srivastava, A.K. and Kumar, M., 2021. Optical Modulation via Coupling of Distributed Semiconductor Heterojunctions in a Si-ITO-Based Subwavelength Grating. Physical Review Applied, 15(5), p.054029, https://doi.org/10.1103/PhysRevApplied.15.054029

[32] Huang, Y.Z., Pan, Z. and Wu, R.H., 1996. Analysis of the optical confinement factor in semiconductor lasers. Journal of applied physics, 79(8), pp.3827-3830, https://doi.org/10.1063/1.361809

[33] Dorodnyy, A., Salamin, Y., Ma, P., Plestina, J.V., Lassaline, N., Mikulik, D., Romero-Gomez, P., i Morral, A.F. and Leuthold, J., 2018. Plasmonic photodetectors. IEEE Journal of Selected Topics in Quantum Electronics, 24(6), pp.1-13, 10.1109/JSTQE.2018.2840339

[34] Kaushik, V., Rajput, S., Srivastav, S., Singh, L., Babu, P., Heidari, E., Ahmed, M., Al-Hadeethi, Y., Dalir, H., Sorger, V.J. and Kumar, M., 2021. On-chip nanophotonic broadband wavelength detector with 2D-Electron gas. Nanophotonics, https://doi.org/10.1515/nanoph-2021-0365

[35] Rajput, S., Kaushik, V., Singh, L., Pandey, S.S.K., Mishra, R.D. and Kumar, M., 2021. Efficient Photodetector Based on Sub-Bandgap Transition in Silicon-ITO Distributed-Heterojunctions. Journal of Lightwave Technology, 39(21), pp.6886-6892, $\underline{10.1109 / J L T .2021 .3106451}$ 
